\title{
Avaliação do protocolo PediaSuit na função motora grossa de pacientes com paralisia cerebral
}

\author{
Evaluation of the PediaSuit protocol on the great motor function of patients with cerebral \\ palsy
}
Evaluación del protocolo PediaSuit sobre la gran función motora del paciente con parális cerebral

Henrique Botelho Carvalho Rodrigues Pinto ${ }^{1 *}$, Juliana de Oliveira Nunes ${ }^{1}$, Bruna Marques Morais ${ }^{2}$, Ricardo Silva Franco ${ }^{2}$, Pedro Henrique Azevedo Matos ${ }^{2}$, Raian Borges Silva ${ }^{2}$, Marcus Vinícius Denis Valle Reis ${ }^{1}$, Lorena Gonçalves Simões ${ }^{2}$, Isabela Rocha Lacerda Oliveira², Camila Gigante Rocha ${ }^{2}$.

\section{RESUMO}

Objetivo: Avaliar os benefícios do protocolo PediaSuit na Função Motora Grossa (GMFM-66) em Pacientes com Paralisia Cerebral (PC). Métodos: Trata-se de uma revisão bibliográfica integrativa, a partir da seleção de 20 artigos, considerando como critérios de inclusão, os estudos que descrevem a aplicação da Terapia Neuromotora Intensiva (TNI) através do protocolo PediaSuit em crianças com PC, com foco na GMFM-66; diagnóstico clínico de paralisia cerebral, realizado entre indivíduos de ambos os sexos; diagnóstico funcional, leve a moderado, nas idades de 3 a 15 anos; protocolo PediaSuit. Tendo em vista tais critérios, utilizou-se, especificamente, 5 artigos, sendo, 1 estudo observacional longitudinal com 22 crianças, 1 estudo transversal com 5 crianças e 3 estudos de caso, com 1 criança. Resultados: $O$ estudo evidenciou que o protocolo PediaSuit apresentou progressos na reabilitação, sobretudo no ajuste contínuo, entre a musculatura agonista e antagonista. Os benefícios favorecem a inervação recíproca, como fator imprescindível para a coordenação motora, ganhos funcionais e aquisições de habilidades. Considerações finais: O PediaSuit apresenta-se como um importante método terapêutico no tratamento de crianças com PC. No entanto, devido à escassez de um maior volume de pesquisas sobre a efetividade, faz-se necessário que novos estudos sejam realizados, para demonstrar sua eficácia.

Palavras-chave: Paralisia cerebral, PediaSuit, Fisioterapia, Reabilitação.

\section{ABSTRACT}

Objective: To evaluate the benefits of the PediaSuit protocol on Gross Motor Function (GMFM-66) in Patients with Cerebral Palsy (CP). Methods: This is an integrative bibliographic review, based on the selection of 20 articles, considering as inclusion criteria, studies that describe the application of Intensive Neuromotor Therapy (TNI) through the PediaSuit protocol in children with CP, focusing on GMFM-66; clinical diagnosis of cerebral palsy, performed between individuals of both sexes; functional diagnosis, mild to moderate, aged 3 to 15 years; PediaSuit protocol. In view of these criteria, 5 articles were used specifically, 1 longitudinal observational study with 22 children, 1 cross-sectional study with 5 children and 3 case studies with 1 child. Results: The study showed that the PediaSuit protocol showed progress in rehabilitation, especially in the continuous adjustment, between the agonist and antagonist muscles. The benefits favor reciprocal innervation, as an essential factor for motor coordination, functional gains and skills acquisition. Final considerations: PediaSuit presents itself as an important therapeutic method in the treatment of children with CP. However, due to the scarcity of a greater volume of research on effectiveness, it is necessary that further studies are carried out, to demonstrate its effectiveness.

Keywords: Cerebral palsy, PediaSuit, Physiotherapy, Rehabilitation.

\section{RESUMEN}

Objetivo: Evaluar los beneficios del protocolo PediaSuit sobre la función motora gruesa (GMFM-66) en pacientes con parálisis cerebral (PC). Métodos: Se trata de una revisión bibliográfica integradora, basada en la selección de 20 artículos, considerando como criterios de inclusión, estudios que describen la aplicación de Terapia Neuromotora Intensiva (TNI) a través del protocolo PediaSuit en niños con PC, con foco en GMFM66; diagnóstico clínico de parálisis cerebral, realizado entre individuos de ambos sexos; diagnóstico funcional, leve a moderado, de 3 a 15 años; Protocolo PediaSuit. Teniendo en cuenta estos criterios, se utilizaron específicamente 5 artículos, 1 estudio observacional longitudinal con 22 niños, 1 estudio transversal con 5

\footnotetext{
${ }^{1}$ Faculdade Santo Agostinho (FASA), Vitória da Conquista - BA. *E-mail: henriquebotellho@gmail.com

2 Faculdade Santo Agostinho (FASA), Itabuna - BA.
} 
niños y 3 estudios de caso con 1 niño. Resultados: El estudio mostró que el protocolo PediaSuit mostró avances en la rehabilitación, especialmente en el ajuste continuo, entre los músculos agonistas y antagonistas. Los beneficios favorecen la inervación recíproca, como factor esencial para la coordinación motora, las ganancias funcionales y la adquisición de habilidades. Consideraciones finales: El PediaSuit se presenta como un método terapéutico importante en el tratamiento de niños con PC. Sin embargo, debido a la escasez de un mayor volumen de investigación sobre efectividad, es necesario que se realicen más estudios, para demostrar su efectividad.

Palabras clave: Parálisis cerebral, PediaSuit, Fisioterapia, Rehabilitación.

\section{INTRODUÇÃO}

A Encefalopatia Crônica não progressiva da infância, comumente chamada de Paralisia Cerebral (PC), é uma série de distúrbios cerebrais crônicos, não progressivos do movimento ou da postura. Foi descrita pela primeira vez em 1843, quando William John Little, um ortopedista inglês, observou e descreveu 47 crianças portadoras de rigidez espástica. Entretanto, o termo PC foi introduzido por Sigmund Freud, enquanto estudava a "Síndrome de Little". Trata-se de uma lesão do sistema nervoso central, ainda em desenvolvimento, que afeta o tônus, os reflexos e compromete o desempenho motor do indivíduo. O diagnóstico abrange síndromes clínicas, em tipo e gravidade, além de uma variedade de comorbidades neurológicas (GLADER L e BARKOUDAH E, 2019).

Entre as causas que provocam a Paralisia Cerebral, a prematuridade é a principal etiologia. A diversidade de quadros clínicos requer que pacientes e familiares, sejam abordados de maneira sistematizada (HAFSTRÖM M, et al., 2018). Estima-se que o número de pessoas com paralisia cerebral, gire em torno de 2,1 casos, para cada 1000 nascidos vivos. Essa incidência mantém-se constante ao longo de décadas. Em países como o Brasil, com grande heterogeneidade regional e desigualdade de acesso aos cuidados de saúde, é possível a convivência de cenários distintos, com a possibilidade de uma maior prevalência de $P C$ nos grupos populacionais, caso fossem adequadamente avaliados (GLADER L e BARKOUDAH H, 2019).

O diagnóstico geralmente é feito entre os 12 e 24 meses de idade, embora tenha sido realizado antes desse período. Não deve ser baseado em uma anormalidade isolada, mas quando o paciente apresentar sinais neurocomportamentais, tais como, excesso de docilidade ou irritabilidade, má alimentação no período neonatal, vômitos com frequência e pouca atenção visual. Além desses sinais, é necessário ficar atento aos reflexos do desenvolvimento, sabe-se que em bebês e crianças com PC, alguns reflexos não são adequadamente integrados ou inibidos, como é o caso dos reflexos motores relacionados à postura. Sendo assim, recomenda-se avaliar o desenvolvimento motor das crianças em todas as consultas de cuidados preventivos pediátricos (GLADER L e BARKOUDAH H, 2019).

Configura-se como fatores para risco de $\mathrm{PC}$, todos os que influenciam negativamente a saúde da mãe, a exposição a agentes tóxicos e infecciosos, as condições de viabilidade e nutrição do bebê, as condições de parto e a ocorrência de eventos hipóxicos ou traumáticos no período perinatal. As condições de maior risco para o desfecho de paralisia cerebral, são a prematuridade abaixo de 28 semanas, o peso do nascimento abaixo de $1500 \mathrm{~g}$, e o índice de vitalidade do recém-nascido, aferido pelo índice de Apgar menor que 7 no quinto minuto. Ademais, entende-se que múltiplos fatores potencializam o dano cerebral (GLADER L e BARKOUDAH H, 2019)

A paralisia cerebral pode ser classificada de acordo com características clínicas, como espasticidade, discinesia e ataxia; distribuição anatômica unilateral e bilateral; classificação da função motora; distúrbios neurológicos, sensoriais, cognitivos, comportamentais, que variam, de acordo com cada indivíduo. Devido à variedade, foi necessário classificar diferentes subtipos de PC. Desse modo, foi criado o Sistema de Classificação da Função Motora Grossa (GMFCS) para pessoas com paralisia cerebral. O sistema avalia atividades motoras diversas que incluem o sentar, transferências e mobilidade. O GMFCS é uma classificação eficiente e eficaz para identificar as diferenças na paralisia cerebral, e, desse modo, obter o planejamento ideal de reabilitação motora (HADJINICOLAOU AD, et al., 2019; HIRATUKA E, et al., 2010).

Diversos tipos de reabilitação para pacientes com paralisia cerebral estão descritos na literatura, como forma de estimular a expectativa e a qualidade de vida dos mesmos. Entre os mais notáveis, destaca-se o Protocolo PediaSuit, que surgiu em meados dos anos 2000, nos Estados Unidos. O Protocolo PediaSuit 
agrega técnicas e métodos, de base fisioterápica intensiva, indicados para o tratamento de Paralisia Cerebral, atrasos de desenvolvimento neuropsicomotor, lesões cerebrais traumáticas, autismo e outras condições que afetam as funções motoras e/ou cognitivas (SILVA CS e LACERDA RV, 2017).

O método realiza o alinhamento biomecânico, pressiona as articulações, reeducando o cérebro através de estimulação de externos receptores e proprioceptores, que enviam informações sensoriais ao sistema nervoso central, para reconhecer padrões de movimentos corretos e atividade muscular apropriada (SILVA CS e LACERDA RV, 2017). Apresenta quatro etapas distintas: aquecimento e alongamento, uso da veste terapêutica, a gaiola do macaco (Monkey Cage), e a gaiola da aranha (Spider Cage). O traje conta com joelheiras e sapatos que são conectados por um sistema de bandas elásticas ajustadas, para aplicar em diferentes graus de tensão e oferecem suporte e resistência para o movimento (BUDTINGER LF e MULLER $A B, 2018)$.

Embora a Paralisia Cerebral tenha sido largamente estudada no mundo inteiro, o processo de reabilitação ainda é uma seara que carece de atenção e discernimento científico. No entanto, aos poucos, ocorrem avanços, sobretudo após a criação de protocolos intensivos como o PediaSuit. O mesmo, como atesta esse estudo, poderá potencializar ganhos motores, melhorar a função motora grossa e o rendimento funcional das crianças com diagnostico de paralisia cerebral. Nesta perspectiva, o processo de reabilitação busca atenuar os efeitos da deficiência e promover a independência. O protocolo PediaSuit apresenta-se como um recurso para o tratamento, visando restabelecer o alinhamento biomecânico e a descarga de peso para a regularização do tônus muscular (BUDTINGER LF e MÜLLER AB, 2018).

O estudo teve como objetivo principal avaliar os benefícios do protocolo PediaSuit na Função Motora Grossa (GMFM-66), em pacientes portadores de Paralisia Cerebral, descrevendo o processo de intervenção e seus resultados, enfatizando a reabilitação da marcha, o controle de tronco e a melhora da função motora grossa. Para tanto, foram selecionados cinco protocolos do Método PediaSuit, aplicados em intervalos de quatro meses.

\section{MÉTODOS}

Trata-se de um trabalho de revisão bibliográfica, de caráter integrativo, do tipo descritivo, a partir de cinco artigos que descrevem a aplicação do protocolo PediaSuit. Esse tipo de revisão consiste em uma busca de estudos e pesquisas sobre determinado assunto, que permita identificar lacunas que podem ser preenchidas mediante outros estudos. A revisão integrativa possibilita uma avaliação crítica e a síntese de evidências disponíveis sobre o tema investigado em seu produto final, proporcionando uma organização do estado atual do conhecimento e reflexões para a implementação de novas intervenções (MOWBRAY PK, et al., 2015).

A coleta de dados realizou-se com base em cinco estudos correspondentes aos períodos de 2010 a 2021 , disponíveis nas plataformas virtuais: Centro Latino Americano e do Caribe de Informação em Ciências da Saúde (BIREME); Scientific Electronic Library Online (SciELO); Google Acadêmico, Literatura Latinoamericana e do Caribe em Ciências da Saúde (LILACS); Coordenação de Aperfeiçoamento de Pessoal de Nível Superior (CAPES).

Os artigos selecionados obedeceram ao seguinte critério de inclusão: ter sido publicado nas línguas inglesa ou portuguesa e abordar a técnica como um recurso de reabilitação fisioterapêutica. Os estudos utilizaram o instrumento Medição da Função Motora Grossa ou Gross Motor Function Measure (GMFM-88), para acompanhamento do desempenho dos pacientes, aplicado antes e após a intervenção com o Protocolo PediaSuit. O GMFM-88 é um teste padronizado, desenvolvido para quantificar a função motora grossa de crianças portadoras de distúrbios neuromotores, particularmente, a paralisia cerebral. Estudos que investigaram as propriedades psicométricas desse teste infantil informaram que o GMFM88 é um instrumento válido e confiável (HARVEY AR, 2017).

Como critérios de inclusão, foram utilizados artigos originais que tratavam sobre a aplicação da Terapia Neuromotora Intensiva nos protocolos PediaSuit em crianças com Paralisia Cerebral, com ênfase no ganho da função motora grossa. Foram incluídos indivíduos de ambos os sexos, com diagnóstico funcional de leve a moderado e Idades de 3 a 15 anos. Para a obtenção dos resultados, foram utilizados os descritores Paralisia Cerebral, PediaSuit, Fisioterapia, Reabilitação, Terapia Neuromotora Intensiva. 
Figura 1 - Critérios para identificação do estudo.

Termos de busca

Bases de Dados

Idiomas

Excluídos

Elegíveis

Tipo de Estudo

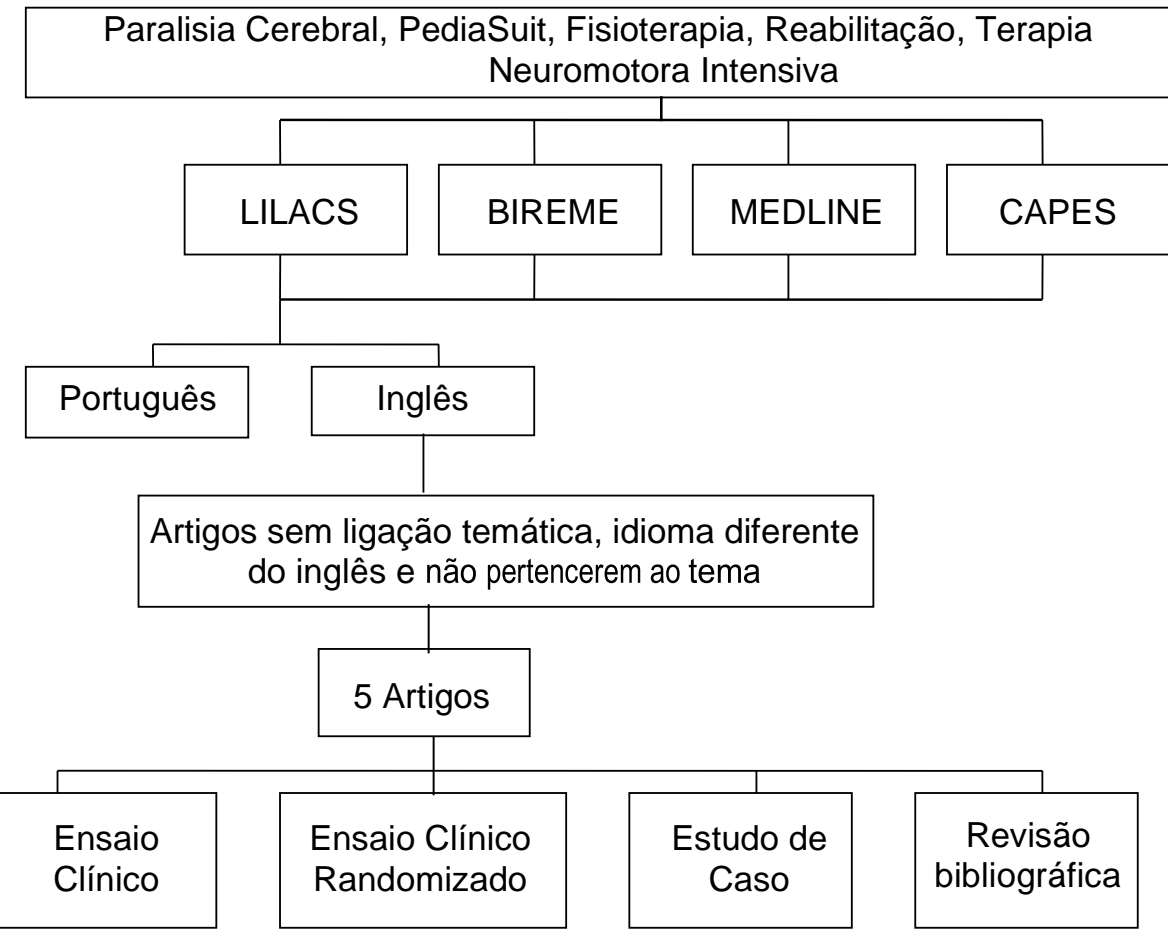

Fonte: Rodrigues HB, et al., 2021.

Para o estudo da amostra e critério de evolução dos indivíduos submetidos ao protocolo PediaSuit, optouse pelo GMFCS, com vistas a avaliar quantitativamente possíveis mudanças na função motora grossa dos participantes do estudo. O Protocolo PediaSuit constitui um período de 4 semanas de tratamento, realizado 5 dias por semana, com 4 horas de duração por sessão/dia. Cada criança estudada realizou 20 sessões, totalizando 80 horas em 4 semanas. As sessões foram acompanhadas pelos profissionais de fisioterapia e terapia ocupacional em dias alternados, sendo 3 sessões de fisioterapia e 2 sessões de terapia ocupacional. Cada sessão de terapia tem a duração de 4 horas por dia.

Os estudiosos são unânimes em afirmar que um dos efeitos da reação neuromotora decorrente da PC é a espasticidade, caracterizada por um quadro de hipertonia muscular, uma resposta de contração do músculo quando ele é estirado de forma rápida, gerando posturas anormais e movimentos alterados. Todas essas alterações podem afetar tanto os membros superiores (MMSS), quanto os membros inferiores (MMII) e tronco. Sendo assim, a melhor forma de acompanhar o desenvolvimento motor do paciente portador de PC é a Medida da Função Motora Grossa (GMFM), considerada um instrumento completo, de fácil aplicação e baixo custo (LEE SH, et al., 2015).

Embora a Paralisia Cerebral tenha sido largamente estudada no mundo inteiro, o processo de reabilitação ainda é uma seara que carece de atenção e discernimento científico. No entanto, aos poucos ocorrem avanços, sobretudo após a criação de protocolos intensivos como o PediaSuit. O mesmo, como atesta esse estudo, é capaz de potencializar ganhos motores, melhorar a função motora grossa e o rendimento funcional das crianças com diagnostico de paralisia cerebral.

O protocolo PediaSuit realizado nos pacientes compreendeu aquecimentos (massagem, cinesioterapia com mobilização ativa e passiva); cinesioterapia (gaiola do macaco, para trabalhar o fortalecimento muscular); cinesioterapia com o uso do traje (gaiola da aranha, utiliza um cinturão preso aos elásticos para realização de exercícios ativos, além de prancha de equilíbrio, bola, cama elástica e rolos); manobras de fisioterapia respiratória; treino de marcha (com uso do suit em terrenos irregulares, escada, rampa, esteira, grama, barras paralelas sem e com obstáculos); atividades de motricidade fina (uso de massinha, desenho com lápis, pintura com pincel, colagem, jogos de encaixe, manipulação de objetos e brinquedos); osteopatia crânio-sacral; e kinesiotape (bandagem funcional) (SILVA CS e LACERDA RV, 2017). 


\section{RESULTADO E DISCUSSÃO}

A terapia com o protocolo PediaSuit, analisada nos respectivos artigos, tem o objetivo de enfatizar a correção postural e o padrão de desenvolvimento. O método se baseia em três princípios: o efeito do macacão ortopédico, a intensidade da terapia, e a participação motora do paciente durante o tratamento (MANGILLI, E. M, 2017). Neste estudo, os cinco artigos são consensuais ao afirmar a viabilidade do tratamento fisioterapêutico com a TNMI, através do protocolo PediaSuit, sendo o mesmo, um recurso terapêutico importante para o desenvolvimento motor de crianças com PC. Os estudos realizados com o protocolo demonstraram uma notável melhoria nas crianças investigadas, focando a análise da função motora grossa através da escala de avaliação GMFM.

Sabe-se que a Terapia Neuromotora Intensiva (TNMI) foca no desenvolvimento motor, no fortalecimento muscular, na resistência, na flexibilidade, na coordenação motora e no equilíbrio, baseando-se em três princípios: a resistência a musculatura; o treino intensivo nas habilidades motoras e a promoção da participação motora ativa. (OLIVEIRA Al, et al., 2017). Nesse sentido, os instrumentos de avaliação utilizados nesta revisão, para a análise dos deficits motores em crianças com PC, realizadas pré e pós intervenção, ocorreram por meio do Sistema de Classificação da Função Motora Grossa (GMFCS); da escala Medida da Função Motora Grossa (GMFM). Segue o quadro descritivo, contendo os objetivos, a metodologia e os principais resultados.

Quadro 1 - Descrição dos estudos e procedimentos utilizados.

\begin{tabular}{|c|c|c|c|c|}
\hline Autor/ano & Título & Objetivos & Metodologia & Resultados principais \\
\hline $\begin{array}{l}\text { Neves EB, } \\
\text { et al. (2013) }\end{array}$ & $\begin{array}{c}\text { Benefícios da Terapia } \\
\text { Neuromotora } \\
\text { Intensiva (TNMI) para } \\
\text { o Controle do Tronco } \\
\text { de Crianças com } \\
\text { Paralisia Cerebral. }\end{array}$ & $\begin{array}{c}\text { Determinar os } \\
\text { ganhos na função } \\
\text { motora de tronco em } \\
22 \text { crianças com PC, } \\
\text { submetidas a cinco } \\
\text { semanas de TNMI } \\
\text { com uso do traje } \\
\text { PediaSuit. }\end{array}$ & $\begin{array}{c}\text { Estudo } \\
\text { observacional } \\
\text { longitudinal de } \\
\text { caráter } \\
\text { quantitativo. }\end{array}$ & $\begin{array}{l}\text { GMFM: diferença para as } \\
\text { dimensões A, B e total } \\
\text { entre Pré e pós TNMI. } \\
\text { - Redução média de } 7,70^{\prime \prime} \\
\text { e } 5,73^{\prime \prime} \text { para o tempo de } \\
\text { oscilação do tronco fora } \\
\text { dos } 4^{\circ} \text { e } 10^{\circ} \text {, e ambos } \\
\text { com } p<0,05 \text {. }\end{array}$ \\
\hline $\begin{array}{l}\text { Oliveira LI, } \\
\text { et al. (2017) }\end{array}$ & $\begin{array}{l}\text { Efetividade do método } \\
\text { suit na função motora } \\
\text { grossa de uma } \\
\text { criança com PC. }\end{array}$ & $\begin{array}{l}\text { Avaliar a efetividade } \\
\text { do método suit na } \\
\text { função motora } \\
\text { grossa. }\end{array}$ & $\begin{array}{c}\text { Estudo } \\
\text { longitudinal de } \\
\text { caso } \\
\text { com } 01 \text { criança } \\
\text { PC }\end{array}$ & $\begin{array}{l}\text { Aumento da capacidade } \\
\text { motora: a) deitar e rolar; } \\
\text { b) sentar; c) engatinhar e } \\
\text { ajoelhar; d) em pé; e) } \\
\text { andar, correr e pular. }\end{array}$ \\
\hline $\begin{array}{l}\text { Maia TA, et } \\
\text { al. (2018) }\end{array}$ & $\begin{array}{l}\text { Avaliação da função } \\
\text { motora grossa em } \\
\text { pacientes com } \\
\text { encefalopatia crônica } \\
\text { não progressiva da } \\
\text { infância com o uso da } \\
\text { suit terapia. }\end{array}$ & $\begin{array}{l}\text { Avaliar a função } \\
\text { motora grossa em } \\
\text { três momentos, sem } \\
\text { o uso da Suit } \\
\text { Terapia, com o uso } \\
\text { parcial e total em } \\
\text { crianças com PC. }\end{array}$ & $\begin{array}{c}\text { Estudo } \\
\text { transversal, com } \\
\text { abordagem } \\
\text { quantitativa em } 5 \\
\text { crianças, com } \\
\text { idade de } 5 \text { a } 11 \\
\text { anos, de ambos } \\
\text { os sexos e com } \\
\text { PC. }\end{array}$ & $\begin{array}{c}\text { Todos obtiveram um } \\
\text { maior escore no GMFM } \\
\text { em todas dimensões, } \\
\text { alcançando um aumento } \\
\text { de até } 23 \% \text { nas } \\
\text { habilidades motoras } \\
\text { grossas. }\end{array}$ \\
\hline $\begin{array}{l}\text { Piovezani } \\
\text { ET, et al. } \\
(2017)\end{array}$ & $\begin{array}{l}\text { Método PediaSuit e a } \\
\text { função motora grossa } \\
\text { de criança com } \\
\text { paralisia cerebral } \\
\text { atáxica. }\end{array}$ & $\begin{array}{l}\text { Verificar a influência } \\
\text { do PediaSuit na } \\
\text { função motora } \\
\text { ampla e na } \\
\text { percepção do } \\
\text { cuidador de uma } \\
\text { criança com PC. }\end{array}$ & $\begin{array}{l}\text { Estudo de caso } \\
\text { com uma criança } \\
\text { de } 6 \text { anos com PC } \\
\text { do tipo atáxica. }\end{array}$ & $\begin{array}{c}\text { Aumento na pontuação } \\
\text { total do GMFM, sendo } \\
\text { mais evidente a dimensão } \\
\text { D }(25,36 \%) \text { após o } \\
\text { tratamento. }\end{array}$ \\
\hline $\begin{array}{l}\text { Silva BM } \\
(2014)\end{array}$ & $\begin{array}{c}\text { Análise } \\
\text { baropodométrica em } \\
\text { criança portadora de } \\
\text { PC submetida a } \\
\text { tratamento com } \\
\text { PediaSuit. }\end{array}$ & $\begin{array}{l}\text { Verificar a influência } \\
\text { do método PediaSuit } \\
\text { na postura e } \\
\text { controle motor por } \\
\text { meio do da } \\
\text { baropodometria. }\end{array}$ & $\begin{array}{c}\text { Estudo de caso } \\
\text { descritivo junto a } \\
\text { uma criança de } \\
\text { três anos com } \\
\text { diagnóstico de } \\
\text { PC. }\end{array}$ & $\begin{array}{c}\text { Melhora ao longo do } \\
\text { tratamento, sobretudo na } \\
\text { distribuição de carga } \\
\text { plantar nos planos de ante } \\
\text { e retro pé. }\end{array}$ \\
\hline
\end{tabular}

Fonte: Rodrigues HB, et al., 2021. 
Os cinco estudos apresentaram consenso quanto a aquisição de ganhos na função motora de crianças portadoras de Paralisia Cerebral através da Terapia Neuromotora Intensiva (TNMI), oferecida pelo protocolo PediSuit. A utilização da Terapia Neuromotora Intensiva (TNMI), compreende a realização de tratamento intensivo durante 3 a 4 horas por dia, por 5 dias por semana, durante 4 a 5 semanas em pacientes que apresentam desordens neuromotoras e cognitivas, resultando em aquisições de habilidades funcionais, em comparação com terapias convencionais. Esses protocolos caracterizam-se pela diversidade de recursos terapêuticos e pela utilização de trajes dinâmicos chamados "suits": um colete, uma bermuda e um sapato adaptado, com gancho e elásticos, utilizados para realizar o alinhamento corporal do paciente (AZEVEDO SS, 2014).

O estudo de Neves EB, et al. (2013), avaliou os ganhos obtidos na função motora de tronco em crianças com diagnóstico de paralisia cerebral, submetidas à cinco semanas de TNMI, com o traje do PediaSuit. A pesquisa foi realizada com 22 crianças de 03 a 08 anos de idade, utilizando-se de dois instrumentos de medida, o GMFM 88 e o sistema de eletrogôniometria wi-fi. O estudo concluiu que a TNMI, associada ao uso do suit (órtese dinâmica), pode promover melhoras significativas no controle de tronco. Os resultados indicaram uma evidente diferença estatística $(p<0,05)$ para as extensões $A$, B e total entre os escores pré e pós TNMI. Neste seguimento a eletrogoniometria wi-fi constatou redução média (pós-Pré) de 7,70"e 5,73"para o tempo de oscilação do tronco fora dos $4^{\circ}$ e $10^{\circ}$, e ambos com $p<0,05$, conforme afirma Neves $E B$, et al. (2013)

Com o intuito de avaliar a efetividade do método PediSuit na função motora grossa, Oliveira LI, et al. (2017), realizaram um estudo de caso junto a uma criança de 6 anos, com paralisia cerebral quadriplégica espástica, classificada no nível III do Gross Motor Classification System Measure, submetida à terapia intensiva PediaSuit durante um mês. Constatou-se, entre a primeira avaliação (antes da intervenção) e a última (término da intervenção), um aumento significativo, em torno de $15 \%$ dos escores das dimensões $D$ (de pé) e E (andar, correr e pular) do GMFM-66. Desse modo, o estudo destacou mudanças longitudinais na função motora grossa.

Numa perspectiva comparativa, Maia TA, et al. (2018), avaliaram a função motora grossa em três momentos: sem o uso da Suit Terapia, com o uso parcial e total da Suit Terapia em 05 crianças portadoras de Encefalopatia Crônica não progressiva da infância, com idade de 5 a 11 anos, de ambos os sexos. Nesse estudo, os resultados atestaram que todos obtiveram maior escore no GMFM em todas dimensões, alcançando um aumento de até $23 \%$ nas habilidades motoras grossas.

Neste estudo, notou-se aumento do desempenho motor grosso das crianças com o uso do protocolo PediaSuit, o que se justifica, segundo a análise dos resultados, pelo fato de que a órtese proporciona uma maior estabilidade e confiança para a criança. No entanto, no entendimento de Frange PC, et al. (2012), diversos estudos reforçam, que mesmo havendo indicações de uma tendência na melhora da função, após o programa intensivo de fisioterapia (PIF), neste caso, através do protocolo PediaSuit, a efetividade na marcha e na função motora, ainda não foi estabelecida. Do mesmo modo, a intensidade apropriada dos exercícios e do alongamento, necessária para maximizar os benefícios, continua desconhecida.

Contrariando essa afirmação, os cinco estudos atestam a eficácia do Protocolo PediaSuit, destacando o aumento progressivo da função motora do grupo que foi submetido ao tratamento com o protocolo e comparado com o grupo controle, sem o uso do traje terapêutico. O instrumento de avaliação utilizado foi o GMFM-66 para mensuração dos valores obtidos. Onde foi visto um aumento significativo em todas as dimensões. No entanto, na compreensão de Rosa PC, et al. (2019), a despeito de estudos atualizados sobre o protocolo PediaSuit associado à TNMI, foram observados, que a maioria conta com um número reduzido de participantes, comprometendo a busca de pesquisas fidedignas.

Ainda no quesito influência do PediaSuit na função motora ampla, Piovezani et al. (2017), realizaram um estudo de caso de uma criança de 6 anos com paralisia cerebral do tipo atáxica. Foram implementadas 20 sessões de PediaSuit, 5 vezes por semana, durante 2 horas diárias, no período de 1 mês. Os resultados desse estudo, apontaram que houve um aumento na pontuação total do GMFM, sendo mais evidente na dimensão D (25,36\%), após o tratamento. Os demais domínios também apresentaram diferenças significativas sendo eles: sentar $10 \%$, engatinhar e ajoelhar $14,3 \%$ e andar, correr e pular $3,5 \%$. 
Do mesmo modo, Silva BM (2014), realizou um estudo de caso junto a uma criança do sexo feminino, de três anos, com diagnóstico de PC. O estudo acompanhou o tratamento durante o período de 4 semanas, 4 horas por dia, 5 vezes por semana. Como instrumento de avaliação foi proposto a escala GMFM e o baropodômetro. Na descrição dos resultados, observou-se que houve uma melhora satisfatória ao longo do tratamento, principalmente na distribuição de carga plantar máxima, nos planos de ante e retro pé.

Como vimos, foram analisados 5 estudos acerca do protocolo PediaSuit, e em todos foi constatado avanços do desempenho da função motora grossa geral, sendo a variação do escore total de 3 a 10 pontos percentuais. As mudanças foram identificadas nas dimensões estudadas, deitar e rolar; sentar; engatinhar e ajoelhar; andar, correr e pular. Houve evidente melhora da postura sentada, na realização de transferências, sem o auxílio das mãos e no equilíbrio estático. Além disso, as crianças apresentaram evolução do equilíbrio dinâmico, adquirindo a marcha independente, a curtas distâncias, o que antes só era possível com apoio (BUDTINGER LF, MÜLLER AB, 2018)

Mediante os resultados apresentados, o estudo constata que o tratamento com o Protocolo PediaSuit consegue potencializar a função motora grossa da criança com paralisia cerebral, ou seja, evidenciou que os variados manejos presentes no protocolo PediaSuit apresentaram benefícios de reabilitação física, demonstrando ganhos potenciais no desempenho; no aspecto motor adaptativo e motor grosso; impulsionou o ajuste contínuo entre a musculatura agonista e a antagonista (HARVEY AR, 2017).

Tais benefícios segundo Possel EP, et al. (2018), favorecem o trabalho de inervação recíproca, sendo este, fator imprescindível para o processo de evolução da coordenação motora e aquisições de novas habilidades. O resultado deste trabalho de revisão foi promissor, afinal, descreveu e analisou os níveis de disfunção motora grossa de crianças com PC sem e com o uso do protocolo PediaSuit, apresentando resultados coerentes, como o ganho de funcionalidade de cada criança em todas as dimensões avaliadas pelo GMFM, sobretudo nas dimensões $D$ e $E$.

É evidente que a iniciativa de novos métodos de reabilitação do controle motor, da postura e do equilíbrio, em disfunções neurológicas e sensoriais, interessa aos profissionais da área de saúde, mas não só. Trata-se de alternativas benéficas, se comprovados os seus resultados e que realmente agreguem valores e qualidade de vida para os portadores de necessidades específicas. Não há outro modo de alcançar êxito, em qualquer protocolo, sem lançar mão de pesquisas na área de reabilitação, independentemente da idade e da doença, de forma profunda e criteriosa. Entretanto, para Rosa PC, et al. (2019), é preciso realizar novas pesquisas apropriadas à patologia para assim comprovar, efetivamente, os benefícios do protocolo proposto.

Na visão de Silva CS e Lacerda RV (2017), uma vez comprovado seus benefícios, os novos métodos serão administrados em pacientes com disfunção neuromotora, que necessitam recuperar a independência funcional e a autoestima. Nesse sentido, a reabilitação é um processo complexo, porém amplo, profundo e desafiador. Através de métodos, como o PediaSuit, por exemplo, torna-se possível uma dinâmica de aprendizado direcionado à obtenção de muitas capacidades (às vezes tidas como perdidas), como o ganho de força muscular, a estabilidade, o controle motor, a restauração do controle postural e o estímulo constante de uma mobilidade independente.

Entre os novos métodos de reabilitação, o Protocolo PediaSuit foi a abordagem terapêutica escolhida por ser a mais recente, ainda em fases iniciais de estudo. Para Neves EB, et al. (2013), o uso de trajes especiais (suits) é que a sua utilização induz um forte estímulo aferente sobre o sistema nervoso, principalmente o proprioceptivo, buscando recuperar o atraso motor decorrente da PC, como se pode notar, no decorrer do estudo. Entretanto, apesar dos avanços, carece de maiores estudos, como forma de comprovar sua eficácia, além de possibilitar um maior entendimento sobre as técnicas utilizadas e suas perspectivas, a curto, médio e longo prazo.

O interesse pela proposta surgiu pela necessidade de buscar novas abordagens terapêuticas no universo de tratamento da paralisia cerebral. Nessa busca, o protocolo suit e sua metodologia, bem como seus principais avanços e desafios, chamaram a atenção para um olhar mais atento, o que só seria possível através de um estudo bibliográfico e uma seleção criteriosa de publicações específicas e atualizadas. No entanto, 0 levantamento teórico na literatura acerca do protocolo PediaSuit associado à TNMI, demonstrou uma visível carência em pesquisas mais robustas, como tem enfatizado Rosa PC, et al. (2019) e outros autores. 
A título de exemplificação, dos 5 estudos incluídos neste trabalho, quatro apresentam um número reduzido de participantes e apenas um estudo apresentou 22 participantes. O volume de casos analisados, não apresenta um número expressivo, embora não inviabilize os estudos ou retire sua credibilidade. Segundo Frange CP, et al. (2012), futuras investigações poderão esclarecer possíveis inconsistências observadas nos resultados dos estudos, provavelmente devido à heterogeneidade metodológica, às diferenças nos tipos de protocolos adotados, às características dos participantes e os instrumentos de avaliação, favorecendo a prática baseada em evidências que demonstrem eficácia do tratamento.

\section{CONSIDERAÇÕES FINAIS}

O estudo teve como objetivo principal avaliar os benefícios do protocolo PediaSuit na Função Motora Grossa (GMFM-66) em Pacientes Portadores de Paralisia Cerebral, verificando sua eficácia e os resultados no processo de reabilitação. Nesta perspectiva, os cinco estudos apresentados demonstram que houve efetividade do protocolo PediaSuit, apresentando influência no desempenho funcional das crianças, destacatando uma melhor performance das habilidades motoras grossas proposta pela GMFM-88. Assim, constatou-se a evidência de benefícios no processo de reabilitação, ainda que de modo escasso (dado ao reduzido número de pacientes estudados), através do protocolo PediaSuit. No entanto, o mesmo apresentase como um importante método terapêutico na reabilitação de crianças com paralisia cerebral.

\section{REFERÊNCIAS}

1. ANDRADE PO, et al. Perfil cognitivo, déficits motores e influência dos facilitadores para reabilitação de crianças com disfunções neurológicas. Revista Paulista de Pediatria, 2011, 29(3): 320-327.

2. AZEVÉDO SS. O efeito do método Therasuit na função motora de uma criança com Paralisia Cerebral. Trabalho de Conclusão de Curso (Graduação em Fisioterapia). Universidade Fernando Pessoa, Porto, 2014. $<$ https://bdigital.ufp.pt/handle/10284/4336>.

3. BUDTINGER LF, MÜLLER AB. Método PediaSuit no tratamento da paralisia cerebral: relato de casos. Revista FisiSenectus, 2018; 6(1): 4-12.

4. DIAS AB, et al. Desempenho funcional de crianças com paralisia cerebral participantes de tratamento multidisciplinar. Revista Fisioterapia e Pesquisa, 2010; 17(3): 225-9.

5. FRANGE CP, et al. Revisão Sistemática do Programa Intensivo de Fisioterapia Utilizando a Vestimenta com Cordas Elásticas. Faculdade de Ciências Médicas de Minas Gerais (FCMMG), Revista Neurociências, 2012, 20(4): 517-526

6. GLADER L, BARKOUDAH H. Paralisia Cerebral: epidemiologia, etiologia e prevenção. Uptodate, 2019, https://www.uptodate.com/contents/cerebral-palsy-clinical-features-and-classification .

7. HADJINICOLAOU AD, et al. Is cerebral plasy changing in high resource settings? Data from the Quebec Cerebral Palsy Registry. Journal of Child Neurology. 2019; 34: 10.

8. HAFSTRÖM M, et al. Cerebral Palsy in Extremely Preterm Infants. Pediatrics, 2018;141(1):20171433. doi:10.1542/peds. 2017-1433

9. HARVEY AR. The Gross Motor Function Measure (GMFM). Journal of physiotherapy. 2017;63(3):187-187

10. HIRATUKA E, et al. Adaptação transcultural para o Brasil do sistema de classificação da função motora grossa (GMFCS). Brazilian Journal of Physical Therapy, 2010; 14(6): 537-544.

11. LEE SH, et al. Gross motor function outcome after intensive rehabilitation in children with bilateral spastic cerebral palsy. Annals of rehabilitation medicine. 2015;39(4):624

12. MAIA TA, et al. Avaliação da função motora grossa em pacientes com encefalopatia crônica não progressiva da infância com o uso da suit terapia. Rev. Fisioterapia Brasil, 2018; 19(5Supl): 33-42.

13. MANGILLI EM. Efeitos musculares do Protocolo PediaSuit®em crianças com paralisia cerebral espástica. Dissertação (Mestrado em Saúde Coletiva). Universidade do Extremo Sul Catarinense, Criciúma, 2017. 53 p.

14. MOWBRAY PK, et al. An integrative review of employee voice: Identifying a common conceptualization and research agenda. Rev International Journal of Management Reviews [Internet]. 2015; 17(3): 382-400.

15. NEVES EB, et al. Benefícios da Terapia Neuromotora Intensiva (TNMI) para o Controle do Tronco de Crianças com Paralisia Cerebral. Centro de Pesquisa Vitória, Curitiba-PR. Rev. Neurociências, 2013; 21(4): 549-555.

16. OLIVEIRA LI, et al. Efetividade do Método Suit na Função Motora Grossa de uma criança com Paralisia Cerebral. Portal de Periódicos Revista Interdisciplinar Ciências Médicas - 2018; 1(2): 15-21.

17. OLIVEIRA Al, et al. Aplicabilidade do Sistema de Classificação da Função Motora Grossa (GMFCS) na paralisia cerebral - revisão da literatura. Arq. Bras. Ciên. Saúde, 2010; 35(3): 220-224.

18. PIOVEZANI JC, et al. Método PediaSuit melhora a função motora grossa de criança com paralisia cerebral atáxica. Rev. ConScientiae Saúde, São Paulo, 2017; 16(1): 131-138.

19. POSSEL EP, et al. A Terapia Neuromotora Intensiva (TNMI) na função motora grossa de crianças com paralisia cerebral. Revista Uniandrade, 2018; 19(2): 53-60

20. ROSA KR, et al. TheraSuit e PediaSuit em crianças com paralisia cerebral. Rev. Ref. Saúde, 2019; 2(3); $102-110$.

21. SILVA BM, et al. Análise baropodométrica em criança portadora de paralisia cerebral submetida a tratamento com a técnica PediaSuit: um estudo de caso. Revista Uniandrade, 2014: 15(1): 07-17.

22. SILVA CS, LACERDA RV. Efeitos do protocolo PediaSuit no tratamento de crianças com Paralisia Cerebral. Revista Multidisciplinar do Nordeste Mineiro, 2017; 2(178): 192-207. 